\title{
Peripheral Ulcerative Keratitis and Necrotizing Scleritis Initiated by Trauma in the Setting of Mixed Cryoglobulinemia
}

\author{
Cameron Campell Johnson $^{\mathrm{a}, \mathrm{b}} \quad$ Derek Hunter Ohlstein $^{\mathrm{a}, \mathrm{b}}$ \\ ${ }^{a}$ University of Florida, Department of Ophthalmology, and ${ }^{b}$ North Florida/ \\ South Georgia Veterans Health System, Gainesville, Fla., USA
}

\section{Key Words}

Scleritis · Peripheral ulcerative keratitis · PUK - Mixed cryoglobulinemia - Hepatitis C

\begin{abstract}
Purpose: To report a case of peripheral ulcerative keratitis and necrotizing scleritis precipitated by trauma in a patient with mixed cryoglobulinemia due to hepatitis $\mathrm{C}$ viral infection.

Methods: Case report and literature review.

Results: A 62-year-old man with a history of mixed cryoglobulinemia developed an episode of necrotizing scleritis and peripheral ulcerative keratitis one month after repair of a traumatic scleral defect with patch grafting. This episode resolved following treatment with high-dose corticosteroids and the patient underwent successful repeat patch grafting along with a free conjunctival autograft. This is the second reported case of necrotizing scleritis and peripheral ulcerative keratitis associated with mixed cryoglobulinemia.

Conclusion: Ophthalmologists should be aware of the association between mixed cryoglobulinemia and necrotizing scleritis/peripheral ulcerative keratitis. Patients with this condition experiencing ocular trauma or undergoing ocular surgery should be monitored closely.
\end{abstract}

\section{Introduction}

Peripheral ulcerative keratitis (PUK) is an inflammatory disease which causes destruction of the peripheral corneal stroma and is associated with an epithelial defect. Non-infectious cases are often associated with systemic collagen vascular disease and vasculitis. The systemic diseases most often associated with autoimmune PUK are 
rheumatoid arthritis, relapsing polychondritis, Wegener's granulomatosis, polyarteritis nodosa, and Churg-Strauss syndrome [1]. It is not uncommon for these patients to have an associated necrotizing scleritis [2]. Below, we report a case of PUK and necrotizing scleritis initiated by trauma in a patient with mixed cryoglobulinemia (MC), a known cause of small blood vessel vasculitis [3]. This is only the second case of PUK with associated scleritis due to cryoglobulinemia that the authors are aware of in the ophthalmic literature, and the first in which an episode was precipitated by trauma.

\section{Case Report}

A 62-year-old man presented with a three week history of sharp pain and redness in his left eye. The pain was first noted after he was cutting tile. The patient's medical history included hepatitis C, porphyria cutanea tarda (PCT), and MC. The patient's past ocular history was significant for an episode of PUK in the left eye which was successfully treated with oral steroids one year prior. On physical exam, visual acuity was $20 / 20$ in both eyes. Slit lamp examination revealed a 1.5 -mm scleral laceration with a fragment of porcelain embedded in the sclera at 3 o'clock. The conjunctiva was noted to be inflamed around the area of the foreign body. The cornea exhibited peripheral thinning without inflammation or an epithelial defect at 5 o'clock, consistent with the prior episode of PUK.

The patient was taken to surgery for removal of the foreign body, which consisted of a $2 \mathrm{~mm} \times$ $2 \mathrm{~mm} \times$ less than $1 \mathrm{~mm}$ piece of porcelain tile. The resultant scleral defect measured $4 \mathrm{~mm}$ long and $2.5 \mathrm{~mm}$ wide and extended through approximately $90 \%$ of the scleral thickness. The surgeon who performed the repair reports that the defect was not a simple laceration, but a crater-like defect due to necrosis of the sclera adjacent to the foreign body. It was therefore unamenable to primary closure. Because the structural integrity of the globe appeared to be compromised, a scleral patch graft was sutured over the defect and the conjunctiva was closed with vicryl running and interrupted sutures.

The patient did well postoperatively and had resolution of his pain by one week after surgery. However, he returned one month after surgery complaining of a constant left sided headache. Slit lamp examination showed a conjunctival defect with partial melting of the underlying patch graft, creating a desiccated depression through which the choroid was visible. The area around the defect had $4+$ injection which did not blanch with phenylephrine and was exquisitely tender. The cornea adjacent to the scleral defect had a $1.5 \mathrm{~mm} \times 1 \mathrm{~mm}$ area of corneal melting (fig. 1 ).

Because infectious scleritis and keratitis could not be excluded, the patient was hospitalized and placed on fortified topical and intravenous antibiotics. Scleral and corneal cultures, a complete blood count, erythrocyte sedimentation rate, anti-nuclear antibody titers, anti-neutrophil cytoplasmic antibody titers, rheumatoid factor, rapid plasma reagent, and chest X-ray were also performed with negative results. After 48 hours with no improvement, treatment with intravenous methylprednisolone $1 \mathrm{mg} / \mathrm{kg}$ was initiated. 24 hours later the patient reported mild improvement. He was discharged on oral prednisone and antibiotics. One week post discharge the patient's scleritis had improved significantly and the PUK had resolved with reepithelialization of the cornea. One month after discharge the eye was quiet enough (fig. 2) to proceed with a repeat scleral patch graft with primary conjunctival closure. Due to the poor quality of the conjunctival tissue around the patch graft, the patient had dehiscence of the conjunctiva which was unsuccessfully treated with resuturing and amniotic membrane transplantation. He eventually required a free conjunctival autograft which achieved good results (fig. 3 ). The oral steroids were slowly tapered without recurrence of the disease.

\section{Discussion}

Cryoglobulins are abnormal antibodies which precipitate from the serum at low temperatures. In the disorder of MC they are composed of monoclonal immunoglobulin $\mathrm{M}$ with rheumatoid factor activity against polyclonal immunoglobulin $\mathrm{G}$ [3]. 
Cryoglobulins act as immune complexes and deposit on the endothelium of small- and medium-size blood vessels causing a vasculitis. These proteins have been found to be produced by a monoclonal expansion of B cells in the liver of patients infected with hepatitis $\mathrm{C}$ virus (HCV). Currently more than $90 \%$ of MC is associated with HCV, while $10-70 \%$ of patients with HCV exhibit MC. The vasculitis caused by MC often affects the skin, peripheral nerves, and kidneys causing palpable purpura, weakness and paresthesia, and membranoproliferative glomerulonephritis, respectively [4].

In the eye, posterior pole manifestations of MC include Purtscher-like retinopathy, central serous chorioretinopathy, and central retinal vein occlusion [5]. In the anterior segment, corneal deposits of cryoglobulins, requiring treatment with phototherapeutic keratectomy have been reported [6]. There have also been reports of scleritis associated with HCV and MC. Ali et al. [7] in 1999 described the first case of scleritis associated with $\mathrm{MC}$ and $\mathrm{HCV}$ in the rheumatologic literature. This patient had previously undiagnosed HCV. Treatment was initiated with oral corticosteroids, but the patient eventually required treatment with cyclophosphamide to achieve control of his disease. The authors postulated that scleritis may have been precipitated by the fact that the anterior eye is approximately $2^{\circ} \mathrm{C}$ cooler than the rest of the body, causing cryoglobulins to precipitate. Another case of scleritis associated with MC was described in the ophthalmic literature in 2008 by Rudrarju et al. [8]. This patient, who was diagnosed with HCV 4 years prior, had isolated non-necrotizing anterior scleritis which was controlled with oral prednisone alone. The only previously published case of PUK associated with HCV and MC was reported by Kedhar et al. [9] in 2007. This patient had $70 \%$ corneal thinning associated with a necrotizing scleritis and required cyclophosphamide to achieve disease resolution.

Like Kedhar's patient, our patient had a history of previous episodes of PUK which were not initially attributed to MC. This is an association of which ophthalmologists should be aware. Unlike Kedhar's patient, our patient's disease was able to be controlled with corticosteroids alone. He also differs from previously reported patients in that he had an episode of severe necrotizing scleritis and PUK precipitated by trauma. There have been reports of noninfectious nodular scleritis following trauma [10] and necrotizing sclerokeratitis has been reported following ocular surgery in patients with underlying vasculitides [11]. However, to our knowledge, this is the first report of a patient with $\mathrm{HCV}$ and MC, but no other connective tissue disease, who developed necrotizing scleritis and PUK after trauma.

A confounding factor in this case is the patient's diagnosis of PCT. PCT is an acquired metabolic disease of heme synthesis which leads to the accumulation of porphyrins in the body, which in turn cause lesions of sun-exposed areas. There have been case reports of scleritis associated with PCT $[12,13]$. However, this is unlikely to be the cause of our patient's scleritis and PUK given that it was initiated by trauma and not sun exposure. Also, subsequent to this episode, he developed mild PUK at the 12o'clock position in his contralateral eye. This location is atypical for disease associated with PCT, which has only been reported nasally and temporally in the sun-exposed interpalpebral area. Additionally, an episode of PUK prior to the current one did not have an associated scleritis. Isolated PUK has not been reported with PCT. Finally, his PCT had been quiescent, with no new skin lesions, in the 6 months prior to his presentation, likely due to treatment with serial phlebotomy. The diagnosis of PCT is 
not surprising in our patient given that $\mathrm{HCV}$ is a risk factor for it, with $69 \%$ of patients with PCT in one study being positive for HCV [14].

Another point to consider is whether the initial surgery was at higher risk for failure due to the inflamed state of the eye when the initial scleral patch graft was placed. There have been several reports of graft necrosis when a homologous scleral graft was used for repair of an eye with active inflammation, due to spread of the inflammatory process to the graft. Given that our patient's scleral defect was only $90 \%$ thickness and had no uveal prolapse, one could argue that a repair which simply covered the defect with conjunctiva may have resulted in less of an inflammatory response. A patch graft could have been performed later, if necessary, once the eye was quiet. The success of his subsequent graft was likely due to the fact that inflammation was controlled prior to the surgery [15].

This case, along with previously reported cases of PUK and necrotizing scleritis associated with MC, suggests that it is prudent for ophthalmologists to note the presence of MC in HCV-positive patients during treatment of ocular trauma and during preoperative evaluation prior to elective surgery. The additional surgical risk, although small, could be discussed with the patient, and the surgeon could monitor the patient more closely postoperatively. This is especially relevant in that it has been shown that early treatment of surgically induced necrotizing sclerokeratitis is essential to preserving vision [11].

\section{Disclosure Statement}

The authors report no conflicts of interest.

\section{Sources of Support}

Funded in part by an unrestricted grant from Research to Prevent Blindness. Publication of this article was funded in part by the University of Florida Open-Access Publishing Fund. 


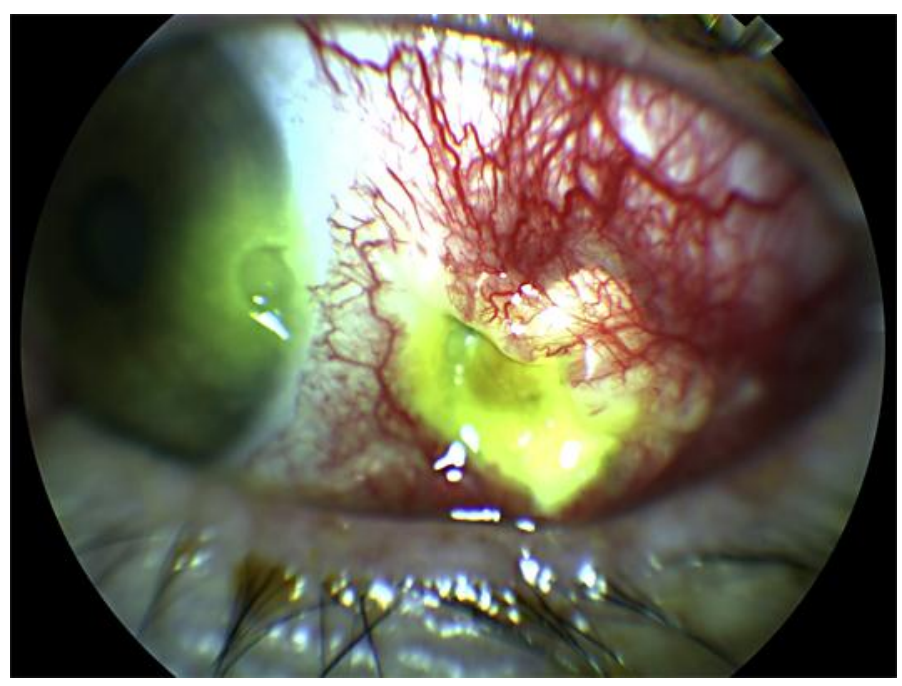

Fig. 1. Necrotizing scleritis and peripheral ulcerative keratitis 1 month after initial surgery to remove foreign body.

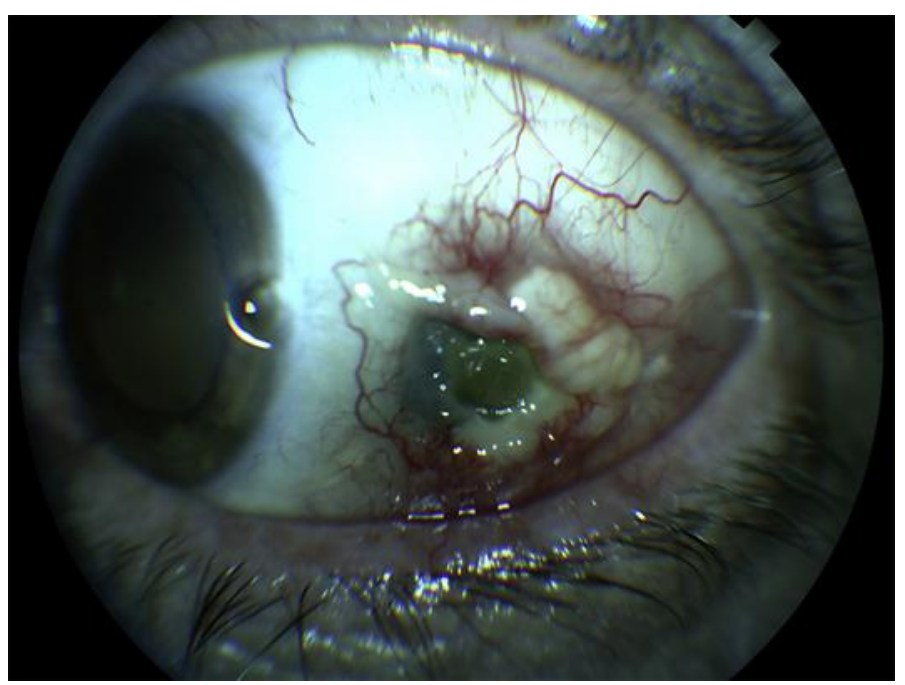

Fig. 2. Eye after treatment with high-dose corticosteroids. 


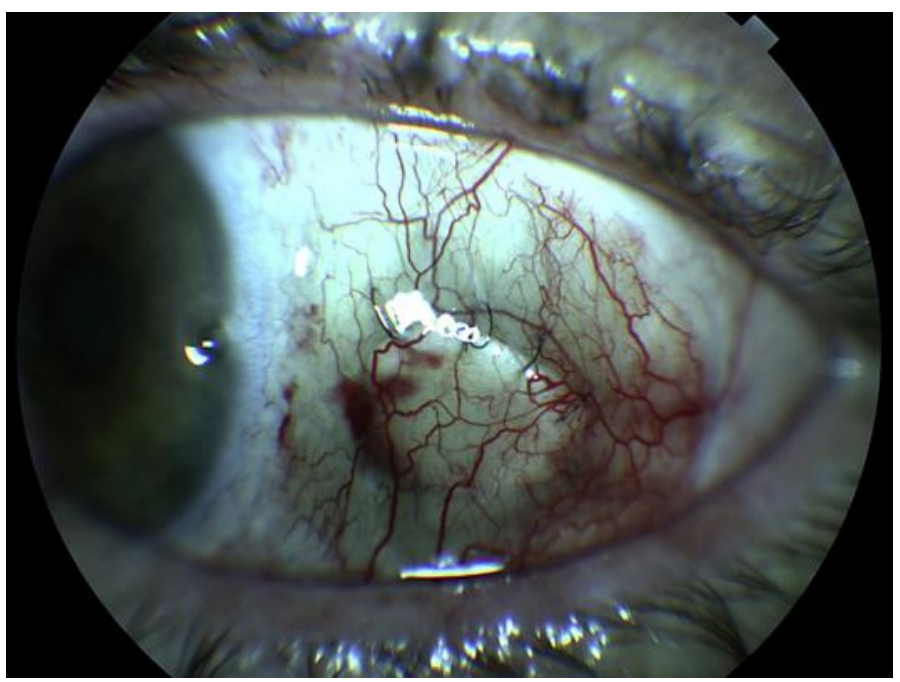

Fig. 3. Eye after scleral patch graft with free conjunctival autograft.

\section{References}

1 Messmer EM, Foster CS: Vasculitic peripheral ulcerative keratitis. Surv Ophthalmol 1999;43:379-396.

2 Stern G: Peripheral corneal disease; in Krachmer JH, Mannis MJ, Holland EJ (eds): Cornea, 2 nd ed. Philadelphia, Elsevier, 2005, pp 339-352.

-3 Iannuzzella F, Vaglio A, Garini G: Management of hepatitis C virus-related mixed cryoglobulinemia. Am J Med 2010;123:400-408.

4 Charles ED, Dustin LB: Hepatitis C virus-induced cryoglobulinemia. Kidney Int 2009;76:818-824.

5 Myers JP, Di Bisceglie AM, Mann ES: Cryoglobulinemia associated with Purtscher-like retinopathy. Am J Ophthalmol 2001;131:802-804.

-6 Perez VL, Chavala SH, Ahmed M, Chu D, Zafirakis P, Baltatzis S, Ocampo V, Foster CS: Ocular manifestations and concepts of systemic vasculitides. Surv Ophthalmol 2004;49:339-418.

7 Ali Y, Ghafouri M, Weitzman M, McLean R, Bonis P: Refractory scleritis in a patient with cryoglobulinemia and hepatitis C. J Clin Rheumatol 1999;5:371-373.

8 Rudraraju M, Singh V, Cobb SH, Douglas D, Dharmendra P, Shen J: Scleritis associated with hepatitis C: a case report and review of literature. Ann Ophthalmol 2008;40:197-199.

-9 Kedhar SR, Belair M, Jun AS, Sulkowski M, Thorne JE: Scleritis and peripheral ulcerative keratitis with hepatitis C virus-related cryoglobulinemia. Arch Ophthalmol 2007;125:852-853.

10 Smith JR, Chee SP: Nodular anterior scleritis associated with ocular trauma. Singapore Med J 2001;42:180.1.

11 O’Donoghue E, Lightman S, Tuft S, Watson P: Surgically induced necrotizing sclerokeratitis (SINS)precipitating factors and response to treatment. Br J Ophthalmol 1992;76:17-21.

12 Altiparmak UD, Oflu Y, Kocaoglu FA, Katircioglu YA, Duman S: Ocular complications in 2 cases with porphyria. Cornea 2008;27:1093-1096.

13 Salmon JF, Strauss PC, Todd G, Murray AD: Acute scleritis in porphyria cutanea tarda. Am J Ophthalmol 1990;109:400-406.

14 Jalil S, Grady JJ, Lee C, Anderson KE: Associations among behavior-related susceptibility factors in porphyria cutanea tarda. Clin Gastroenterol Hepatol 2010;8:297-302.

15 Hamill MB: Management of scleral perforation; in Krachmer JH, Mannis MJ, Holland EJ (eds): Cornea, 2nd ed. Philadelphia, Elsevier, 2005 pp 1863-1870. 